\title{
Applications of Kramers Escape Rate Theory With \\ Power-Law Distributions
}

\author{
Yanjun Zhou
}

Kramers escape rate theory is the most important one of modern reaction rate theories. However, one key assumption of the theory that thermodynamic equilibrium must prevail throughout the entire system studied is farfetched for open complex systems. Thereby, Kramers escape rates are generalized to describe rates of reactions in nonequilibrium systems with power-law distributions. Kramers escape rates in the very low damping systems, in overdamped systems and in the low-to-intermediate damping (LID) systems are investigated and the corresponding escape rates are obtained respectively on the basis of nonextensive statistics. When apply to biological, physical and chemical systems in each damping systems, these generalized escape rates with power-law distribution show a better agreement with experimental rates as compared with the traditional Kramers escape rates. It is expected that the generalized result can lead to an insight into the research on reaction rate theory for nonequilibrium complex systems with power-law distributions.

Keywords: Kramers escape rate theory; Power-law distribution 


\section{Introdução}

Kramers escape rate theory, a theoretical reaction model, which describes the thermal escape of a Brownian particle out of a metastable well, has been got high interests in biology, physics and chemistry $[1,2]$ etc. In 1940, Kramers realized a very unsatisfactory feature of the transition state theory (TST), whose escape rate is in contradiction to the fluctuation-dissipation theorem. So he defined a prefactor $\mu$ to remedy this defect [3]. He yielded three explicit formulas of $\mu$ for the escape rate in a very low damping, intermediate and overdamped cases respectively according to different dissipative coupling to the bath. His outstanding results point new ways to understand the dynamic features of systems.

When Brownian particles move in different damping media, Kramers postulated that the systems can be thermal equilibrium both in the reaction state and product state, a Maxwell-Boltzmann (MB) distribution always holds in the whole time, and any disturbance to the MB distribution can almost be negligible [3]. Under this key assumption, the escape rates in different damping systems are obtained. However, the assumption is unsatisfying in open complex systems. Nonequilibrium is the main feature for open complex systems. As a matter of fact, lots of experimental observations on complex systems have shown non-MB distributions or power-law distributions, such as folding of proteins [4], trapped ion reactions [5], chemical kinetics, and biological and ecological population dynamics [6, 7], reaction-diffusion processes [8], chemical reactions [9], astrophysical and space plasmas [10], etc. The forms of such power-law distributions in various systems include the generalized Lorentzian distributions in the solar wind and space plasmas [11, 12], the $q$-distributions in complex systems within nonextensive statistical mechanics [13], and $\alpha$-distributions appeared in physics, chemistry and elsewhere like $P(E) \sim E^{-\alpha}$ with an index $\alpha>0[5,8,9]$. These power-law distributions may lead to anomalous processes different from those in the realm governed by Boltzmann-Gibbs statistics with MB distribution. At the same time, a class of statistical mechanical theories studying the power-law distributions in complex systems has been constructed, for instance, by generalizing Boltzmann entropy to Tsallis entropy
[13], by generalizing Gibbsian theory [14] to a system away from thermal equilibrium, and so forth. Therefore, Kramers escape rate can be reconsidered under the condition of power-law distributions in complex systems.

\section{Fundamentals}

There are two main theories to calculate the escape rate constants $k$, one is the first passage time theory [ 15 , $16]$ and the other is flux over population theory $[3,16]$. The first passage time is the first time that the particles leave the boundary of $V$. Because of the noise, even the same initial positions will lead to different first passage times, hence the mean first passage time (MFPT) is introduced. If initial positions of the particles are located in the reactant domain, and the finite space $V$ is the domain for the reaction, then the transition from the reactant to the boundary may be characterized by a rate which is simply determined by the inverse MFPT, i.e. $k=1 / \tau$ in very low damping and $k=1 / 2 \tau$ in high damping. Suppose the particle has the initial position $\mathrm{x}_{0}$ or position $\mathrm{x}_{0}$ and momentum $\mathrm{p}_{0}$, and locates in the finite space $V$ with absorbing boundary. If $P(x, t)$ or $P(x, p, t)$ is the probability distribution that has not left by time $t$, then it satisfies the Smoluchowski equation in overdamped system and Klein-Kramers equation in a very low and intermediate damping systems. The corresponding initial conditions and absorbing boundary conditions are $\mathrm{P}(\mathrm{x}, 0)=\delta\left(\mathrm{x}-\mathrm{x}_{0}\right), \mathrm{P}(\mathrm{x}, \mathrm{t})=0$ on $\partial \mathrm{V}$ and $\mathrm{P}(\mathrm{x}, \mathrm{p}, 0)=\delta\left(\mathrm{x}-\mathrm{x}_{0}\right) \delta\left(\mathrm{p}-\mathrm{p}_{0}\right), \mathrm{P}(\mathrm{x}, \mathrm{p}, \mathrm{t})=0$ on $\partial \mathrm{V}$. Introducing the Fokker-Planck operator and adjoint operator, operating on the MFPT with it and doing the time integral, the MFPT is then determined by correspondingly solving the inhomogeneous adjoint equation $[3,16]$,

$$
\begin{gathered}
\frac{p}{m} \frac{\partial \tau(x, p)}{\partial x}-\left(\frac{d U}{d x}+\gamma p\right) \frac{\partial \tau(x, p)}{\partial p}+\frac{\partial}{\partial p}\left(D \frac{\partial}{\partial p} \tau(x, p)\right)=-1, \\
\tau(x, p)=0 \text { on } \partial V, \\
-(m \gamma)^{-1} \frac{d U}{d x} \frac{\partial \tau(x)}{\partial x}+(m \gamma)^{-1} \frac{\partial}{\partial x} D(x) \frac{\partial \tau(x)}{\partial x}=-1, \\
\tau(x)=0 \text { on } \partial V,
\end{gathered}
$$


where $m$ is the mass of the particle, $U$ is a potential field, $\gamma$ is the friction coefficient, $\mathrm{D}$ is the diffusion coefficient. Eqs. (1a)-(2b) are the starting point of the calculations of escape rate constants. For the flux over population theory, if the steady-state current $\mathrm{J}$ and the (nonequilibrium) population inside the initial domain $\mathrm{n}$ are got, the rate of escape $\mathrm{k}$ is then given by the ratio $\mathrm{k}=\mathrm{J} / \mathrm{n}$.

In very low damping systems, Brownian force causes only a tiny perturbation in the undamped energy during one oscillation in the well, therefore the energy is a slowly varying quantity and the phase a fast-varying quantity. So the original Klein-Kramers equation in the canonical variables $(x, p)$ can be written as a diffusion equation in the energy (E) and phase (w). Average the density over the fast phase variable, and an energy diffusion equation in the slow (almost conserved) energy variable is obtained [3]. The chain rule is used to transform Eq. (1a) into the functions of the energy $\mathrm{E}$ and the phase $\mathrm{w}$, average the Eq. (1a) over the phase w and utilize the definition of the time average and action I, i.e. , then Eq. (1a) becomes,

$\left(\frac{I}{m I^{\prime}} \frac{\partial D}{\partial E}-\gamma(E) \frac{I}{I^{\prime}}+\frac{D}{m}\right) \frac{\partial \tau(E)}{\partial E}+D(E) \frac{I}{m I^{\prime}} \frac{\partial^{2} \tau(E)}{\partial E^{2}}=-1$,

where . Eq. (3) is the general energy-diffusion equation of the MFPT or escape rate constant in very low damping system which can both describe the thermal equilibrium and nonequilibrium systems. For intermediate and high damping systems, the rate constants can be got directly by solving Eq. (1) and (2) with absorbing boundary conditions.

\section{Applications}

In the very low damping system, generalized fluctuation-dissipation relation [17] is $\mathrm{D}=\mathrm{m} \gamma \beta^{-1}(1$ $\kappa \gamma \beta$-E), where $\beta=1 / k_{B} T, k_{B}$ is the Boltzmann constant, $\mathrm{T}$ is the temperature, $\kappa$ is a parameter and $\kappa \neq 0$ measures a distance away from thermal equilibrium. The solution to Eq. (3) is

$$
k^{-1}=\tau(E)=\int_{E}^{E_{s}} \frac{\beta}{E^{\prime}} \frac{d E^{\prime}}{\gamma\left(E^{\prime}\right)\left(1-\kappa \beta E^{\prime}\right)^{(1+\kappa) / \kappa}} \int_{0}^{E^{\prime}} d E^{\prime \prime}\left(1-\kappa \beta E^{\prime \prime}\right)^{1 / \kappa},
$$

and this generalized result can well apply to the Josephson junction. The Josephson junction consists of two superconductors coupled by a weak link and it has received much attention in both theory and experiment $[3,18-20]$. At temperatures sufficiently close to the transition temperature, thermal fluctuations can disrupt the coupling of the phases of the order parameters of two superconductors separated by a thin insulating barrier. The Josephson current thereby acquires a noise voltage with a nonzero average value [20]. Generation of a noise voltage with a nonzero average value can be considered that a Brownian particle performing its motion in a potential energy with the damping coefficient and the random force escapes from a metastable state, so it can be directly treated by Kramers escape theory. Since the escape process is stochastic and the bias current of each escape is also stochastic, which result in the distribution function $f(I)$ measured in the experiments. According to the relationship between the distribution function and MFPT [19], the MFPT can be indirectly obtained and testified [21]. Since $\kappa \neq 0$ reflects influence of the environment on the system, $\kappa<0$ and $\kappa>0$ stand for stronger and weaker influence from the environment, which are analogous to superdiffusive effect and subdiffusive effect in nonlinear diffusive media, so $\kappa$ may be considered as an anomalous escape factor.

In the low to intermediate damping (LID) system, the escape rate is obtained based on the flux over population theory [22],

$$
k=\frac{\gamma_{C} I_{C} \beta(1-\kappa)(\kappa+1)\left[\sqrt{1+\frac{4 \alpha\left(1-\kappa \beta E_{C}\right)}{I_{C} \gamma_{C} \beta}}-1\right.}{\sqrt{1+\frac{4 \alpha\left(1-\kappa \beta E_{C}\right)}{I_{C} \gamma_{C} \beta}}+2(1-\kappa)\left(1-\kappa \beta E_{C}\right)-1} k_{\kappa-I S T},
$$


where $\gamma_{c}$ and $I_{c}$ are the friction coefficient and action in the saddle point, factor $\alpha$ is a constant of order unit and $\mathrm{k}_{\mathrm{k} \text {-TST }}$ is the TST rate with power-law distribution [23]. Then we apply our result to the experiment. K.Hara et al. [24] studied the Kramers turnover behavior for the excited-state isomerization of 2-alkenylanthracene in alkane at the high pressure. The experimental material was 2-(2-propenyl) anthracene (22PA), synthesized using the method of Stolka et al. [25] and purified by TLC. Steady-state and time-resolved fluorescence spectra in supercritical (SC) ethane (99.95\%) and SC CO2 $(99.999 \%)$ were measured at $323 \mathrm{~K}$ and at pressures up to 15.1 and 17.4 MPa respectively [24]. The interaction (i.e. dynamic solvent effect) between the solute and solvent is studied and the consequence can be well explained by our LID result. Parameters we adopt in the Eq. (5) keep the same with the experimental data [24]. At the turning point, our result $\mathrm{k} / \mathrm{k}_{\mathrm{TST}}=0.64$ with the power-law parameter $\kappa=-0.28$ agrees with the experimental turning point value $\kappa_{\max }=0.64$. So, it is concluded that our theory represents excellently the experimental result compared to the traditional theory.

In the overdamped system, the escape rate $k$ is [26],

$$
k=\frac{1}{2 \tau(x)}=\frac{1}{2 m \gamma \int_{x}^{b} d y e^{\phi(y)} / D(y) \int_{a}^{y} d z e^{-\phi(z)}}
$$

where $\phi=-\operatorname{In}(1-\kappa \beta U)^{1 / \kappa}$. There exist plenty of real systems describing the very high damping condition, such as single-molecule pulling experiments in biology [27-29]. The pulling of molecular can be considered as an escape process of crossing the barrier. Since the escape process is stochastic and the external force of each escape is also stochastic, which result in the distribution function $f(F)$ measured in the experiment. According to the relationship between $f(F)$ and escape rate $k[28,19]$ as well as the $\mathrm{k}$ and $k_{0}$ (the intrinsic rate in the absence of external forces), other parameters by fitting the observed distribution function $f(F)$ are obtained, such as $\mathrm{k} 0$, the transition state position etc. In the pulling experiment of unfolding of titin, the material is a recombinant construct of 8 tethered I27 titin molecules, and the unfolding of multiple covalently linked proteins can be treated as the unfolding of a single protein with an average effective pulling spring constant. The apparent rate is roughly between one and eight times than the intrinsic rate $\mathrm{k} 0$ of a single titin, and the specific value of $k_{0}$ is confirmed according to the rupture event of molecules in the experiment [29]. We take experimental parameters into Eq. (6) and get that the theoretical result of $k_{0}$ has a good agreement with the experimental one when power-law parameter $\kappa$ adopts a specific value. Thereby, we show an expected result obtained by our generalized expression of the escape rate.

\section{Conclusion}

It should be noticed that nonextensive statistics is the first, but probably not the only, new possible classical statistics for nonextensive systems. Nonextensive systems may obey another statistics other than that of BG or Tsallis, depending on the nature of their underlying dynamics. According to the present studies, nonextensive statistics has been proved to be very useful for the variety nonequilibrium and nonextensive systems and can represent different complex phenomena. Further developments and applications of nonextensive statistics have been continuing.

Based on the nonextensive statistics, we have derived the generalized expressions of the escape rate constants in three different damping systems with power-law distributions according to the MFPT theory and flux over population theory and apply them into different domains. The new results have been proved that our theoretical rates with power-law distribution show good conformity with experimental ones. The advantage of our result with power-law distributions lies in its theoretical foundations and physical meanings, so it is expected that the generalized results can lead to an insight into the research on reaction rate theory for nonequilibrium complex systems with power-law distributions and have wide applications in the further.

\section{Reference}

1. Gardiner, C. W., Handbooks of stochastic methods for physics, 
chemistry and the Natural Sciences, Springer, 2004.

2. Chatterjee, D., Cherayil, B. J., J. Chem. Phys. 2010, 132, 025103.

3. Coffey, W. T., Kalmykov, Y. P., Waldron, J. T., The Langevin Equation: With Applications to Stochastic Problems in Physics Chemistry and Electrical Engineering, Singapore: World Scientific, 2004.

4. Frauenfelder, H., Sligar, S. G. Wolynes, P. G., Science, 1991, 254, 1598.

5. DeVoe, R. G., Phys. Rev. Lett., 2009,102, 063001.

6. Aquilanti, V., Mundim, K. C., Elango, M., Kleijn, S., Kasai, T., Chem. Phys. Lett., 2010, 498, 209.

7. Niven, R. K., Chem.Eng.Sci., 2006, 61, 3785.

8. Gallos, L. K., Argyrakis, P., Phys. Rev. E., 2005, 72, 017101.

9. Claycomb, J. R., Nawarathna, D., Vajrala, V., Miller, J. H. Jr, J. Chem. Phys. 2004, 121, 12428.

10. Scudder, J. D., Astrophys. J., 1994, 427, 446.

11. Treumann, R. A., Jaroschek, C. H. Scholer, M., Phys. Plasmas, 2004, 11, 1317.

12. Collier, M. R., Roberts, A., Vinas, A., Adv. Space Res. 2008, 41, 1704.

13. Tsallis, C., Introduction to Nonextensive Statistical Mechanics: Approaching a Complex World, New York: Springer, 2009.

14. Treumann, R. A. Jaroschek, C. H., Phys.Rev.Lett., 2008, 100, 155005

15. Zwanzig, R., Nonequilibrium Statistical Mechanics, Oxford University Press, New York, 2001.

16. Hanggi, P., Talkner, P.,Borkovec, M., Rev. Mod. Phys. 1990, 62, 251.

17. Du, J. L., J. Stat. Mech., 2012, P02006.

18. Mazo, J. J., Naranjo, F, Zueco, D., Phys. Rev. B., 2010, 82, 094505 .

19. Barone, A., Cristiano, R., Silvestrini, P., J. Appl. Phys., 1985, 58, 3822 .

20. Ambegaokar, V., Halperin, B. I., Phys. Rev. Lett., 1969, 22, 1326.

21. Zhou, Y. J., Du, J. L., J. Stat.Mech., 2013, P11005.

22. Zhou, Y. J., Du, J. L., Physica A, 2014, 403, 244.

23. Du, J. L., Physica A, 2012, 391,1718.

24. Hara, K., Ito, N., Kajimoto, O., J. Chem. Phys., 1999, 110, 1662.

25. Stolka, M., Yanus, J. F., Pearson, J. M., Macromolecules, 1976, 9, 710 .

26. Zhou, Y. J., Du, J. L., Physica A, 2014, 402, 299.

27. Luccioli, S., Imparato, A., Mitternacht, S., Irbäck, A., Torcini, A., Phys. Rev. E., 2010, 81, 010902(R).
28. Dudko, O. K., Hummer, G., Szabo, A., Phys. Rev. Lett., 2006, 96, 108101.

29. Hummer, G., Szabo, A., Biophys. J., 2003, 85, 5.

\section{Yanjun Zhou*}

Unidade Universitária de Ciências Exatas e Tecnológicas, Universidade Estadual de Goiás, Anápolis, GO, Brazil

*Email: yanjunzhou@aliyun.com 